\title{
The monounsaturated fatty acid oleate is the major physiological toxic free fatty acid for human beta cells
}

T Plötz ${ }^{1,2}$, B Krümmel $\mathbb{D}^{2}$, A Laporte ${ }^{1,3}$, A Pingitore $\mathbb{C}^{4}$, SJ Persaud ${ }^{4}$, A Jörns ${ }^{1}$, M Elsner ${ }^{1}$, I Mehmeti ${ }^{1}$ and S Lenzen ${ }^{1,2}$

\begin{abstract}
Free fatty acids (FFAs) can cause glucose intolerance and diabetes. Lipotoxicity to the pancreatic beta cells is considered to be a major underlying cause for this phenomenon. The aim of this study was to analyse the toxicity profile of FFAs in the human EndoC- $\mathrm{BH} 1$ beta-cell line and to compare the results with isolated rat and human islets with special reference to the physiologically most prevalent FFAs palmitic acid (PA) and oleic acid (OA). Toxicity after a 2-day incubation with the different FFAs was analysed by the caspase-3 assay and confirmed by the propidium iodide and annexin $V$ staining tests. The long-chain saturated PA (C16:0) and the monounsaturated OA (C18:1) were both toxic to human EndoC- $\mathrm{BH} 1$ beta cells and pseudoislets, as well as to rat islets, and, as confirmed in a pilot experiment, also to human islets. Furthermore, OA provided no protection against the toxicity of PA. Likewise, elaidic acid (EA, the trans isomer of $\mathrm{OA}$; trans-OA) was significantly toxic, in contrast to the non-metabolisable analogues methylated PA (MePA) and methylated OA (MeOA). Fatty acids with a chain length < $\mathrm{C} 16$ were not toxic in EndoC- $\beta \mathrm{H} 1$ beta cells. Caspase-3 was also activated by linoleic acid (LA)(C18:2) but not by $\gamma$-linolenic acid ( $\gamma$-LNA)(C18:3). Overall, only longchain FFAs with chain lengths $>\mathrm{C} 14$, which generate hydrogen peroxide in the peroxisomal beta-oxidation, were toxic. This conclusion is also supported by the toxicity of the branched-chain FFA pristanic acid, which is exclusively metabolised in the peroxisomal beta-oxidation. The lack of a protective effect of the monounsaturated fatty acid OA has important consequences for a beta-cell protective lipid composition of a diet. A cardioprotective diet with a high OA content does not fulfil this requirement.
\end{abstract}

\section{Introduction}

There is a firm belief in the general population and in the medical profession that a Mediterranean diet reduces the incidence of cardiovascular disease $\mathrm{e}^{1,2}$. This has been thought to be the explanation for the decreasing rate of coronary heart disease mortality in many populations ${ }^{1,2}$. This conviction originates from early epidemiological studies $^{3}$, a view that has been maintained over many decades $^{4,5}$. The high content of the monounsaturated fatty

\footnotetext{
Correspondence: S Lenzen (lenzen.sigurd@mh-hannover.de)

${ }^{1}$ Institute of Clinical Biochemistry, Hannover Medical School, Hannover, Germany

${ }^{2}$ Institute of Experimental Diabetes Research, Hannover Medical School,

Hannover, Germany

Full list of author information is available at the end of the article
}

acid (MUFA) oleic acid (OA) in olive oil is typically thought to be crucially responsible for this cardioprotective effect when compared with diets containing oils with a dominant content of saturated fatty acids (SFAs) ${ }^{1,4,6}$. This concept has had considerable influence on public health policy recommendations regarding fat content and composition of the daily diet.

Central to this concept is the promotion of the consumption of MUFAs as a more healthy substitute for SFAs in the daily diet by the American Heart Association, as well as the US Food and Drug Administration and by major learned societies (https://www.guideline.gov/ summaries/summary/3127). Diets with high MUFA contents have also been considered favourable in counteracting diabetes development ${ }^{7}$, although evidence has never been unequivocal ${ }^{8}$. 
A critical review of the International Nutrition Guidelines for Diabetes comprising an evaluation of the recommendations of six learned international scientific societies by Katsilambros et al. for the Diabetes and Nutrition Study Group of the European Association for the Study of Diabetes (http://www.dnsg-easd.org/info/general/Document/get/18/ documentId/KATSILAMBROS\%20et\%20al.pdf) revealed a unified recommendation for a low intake of SFAs and a minimisation of trans fatty acid intake along with a clear preference for MUFA consumption. This recommendation has been reiterated recently in the Guidelines of the European Society of Cardiology (ESC), which was developed in collaboration with the European Association for the Study of Diabetes (EASD) (https://www.escardio.org/ static_file/Escardio/Guidelines/publications/DIABETWeb_ EM_Diabetes_2013.pdf).

Nevertheless, doubts have been developing during recent years regarding the validity of this concept ${ }^{1,6,9}$, because a number of basic studies failed to confirm the protective effect of MUFAs against SFA-promoted atherogenesis $^{1,6}$. In particular, a cardioprotective effect of OA, representing the principal MUFA component of olive oil, could not be documented ${ }^{1,6}$. Similar doubts have been expressed with respect to an antidiabetic effect of MUFAs ${ }^{8}$.

An antilipotoxic effect of OA and other MUFAs has been documented in many studies on insulin-secreting cell lines of rodent origin ${ }^{10}$. Whether this also applies to human pancreatic beta-cell lines, however, is unknown.

In the present study, we address this question, making use of the new human EndoC- $\beta \mathrm{H} 1$ beta-cell line and for comparison of isolated rat islets, as well as of isolated human islets in a pilot analysis. We show in this study that the MUFA OA is not protective against PA-mediated toxicity to human beta cells. Rather, this main dietary MUFA turned out to be as toxic as PA, the main dietary SFA in humans ${ }^{11,12}$.

This observation is likely to have significant impact on future recommendations for a healthy diet composition with respect to type 2 diabetes (T2DM) prevention, with the aim of counteracting the worldwide rapidly increasing T2DM incidence (http://www.diabetesatlas. org/resources/2017-atlas.html).

\section{Materials and methods \\ Chemicals and media}

Free fatty acids (FFAs) were purchased from SigmaAldrich (Munich, Germany). Cell culture medium (Dulbecco's modified Eagle's medium (DMEM); Life Technologies, Darmstadt, Germany) was supplemented with $5.5 \mathrm{mM}$ glucose, $2 \%$ bovine serum albumin (BSA; Serologicals Proteins Inc, Kankakee, IL, USA), 1\% penicillin/streptomycin (Biochrom, Berlin, Germany), $50 \mu \mathrm{M}$ 2-mercaptoethanol, $10 \mathrm{mM}$ nicotinamide, $5.5 \mu \mathrm{g} / \mathrm{ml}$ transferrin, $6.7 \mathrm{ng} / \mathrm{ml}$ sodium selenite (all from SigmaAldrich). FFA free BSA was from Serva (Heidelberg, Germany).

\section{Human EndoC- $\beta \mathrm{H} 1$ beta-cell line as well as rat and human islet culture}

EndoC- $\beta \mathrm{H} 1$ beta cells (ENDOCELLS SARL, Paris, France) were cultured ${ }^{13}$ and pseudoislets (PIs) were generated and cultured under the same conditions as described previously ${ }^{13}$. Rat pancreatic islets were isolated from six different $250-300 \mathrm{~g}$ adult male Lewis rats by collagenase digestion, separated by Ficoll gradient and handpicked under a stereo microscope ${ }^{10}$. Human islets were isolated at the King's College Hospital Islet Transplantation Unit, with appropriate ethical approval (LREC 01-082 $)^{14}$. The human islets used in this study were isolated from the pancreas of a 49-year-old non-diabetic male donor (body mass index (BMI): $30.3 \mathrm{~kg} / \mathrm{m}^{2}$; blood glucose: $5.3 \mathrm{mM}$ ). Islets were maintained in Connaught Medical Research Laboratories (CMRL) medium supplemented with $2 \%$ human albumin, $4 \mathrm{mM}$ glutamine, $2 \mathrm{mM}$ HEPES (pH 7.2-7.4) and $10 \mathrm{mM}$ nicotinamide at $37^{\circ} \mathrm{C}$, $5 \% \mathrm{CO}_{2}$ prior to functional analyses. FFA stock solutions $(50 \mathrm{mM})$ were prepared in $90 \%$ ethanol. Incubation medium in the final concentrations was prepared using DMEM medium with fatty acid-free $\mathrm{BSA}^{10}$.

\section{Toxicity analyses}

Groups of 30000 human EndoC- $\beta \mathrm{H} 1$ beta cells, 50 human EndoC- $\beta$ H1 PIs, 10 rat islets or 5 human islets, cultured on 96-well plates, were incubated for 2 days with the various FFAs. Caspase- 3 activity was quantified by the CaspaseGlo-3/7 kit (Promega, Mannheim, Germany). Propidium iodide $(5 \mu \mathrm{g} / \mathrm{ml})$ (Sigma-Aldrich) staining was quantified after a $15-\mathrm{min}$ incubation at $37^{\circ} \mathrm{C}$ with the dye by flow cytometry in the FL-3 channel as described before $^{13}$. Annexin $\mathrm{V}$ staining was quantified after a 15 -min incubation at $37^{\circ} \mathrm{C}$ with the dye by fluorescence microscopy using the Annexin V-FITC apoptosis kit (Thermo Fisher Scientific, Waltham, MA, USA). Apoptotic EndoC- $\beta \mathrm{H} 1$ beta-cell nuclei were identified by electron microscopy as described ${ }^{10}$.

\section{Statistical analysis}

Data are expressed as means \pm SEM. Statistical analyses were performed using analysis of variance plus Dunnett's multiple comparison test and Student's $t$-test, unless stated otherwise (Graphpad, San Diego, CA, USA).

\section{Results}

Caspase- 3 was significantly activated in human EndoC$\beta \mathrm{H} 1$ beta cells (Fig. 1a), human EndoC- $\beta \mathrm{H} 1$ PIs (Fig. 1d), isolated rat pancreatic islets (Fig. 1e) and, in a pilot study, isolated human pancreatic islets (Fig. 1f) after a 2-day 

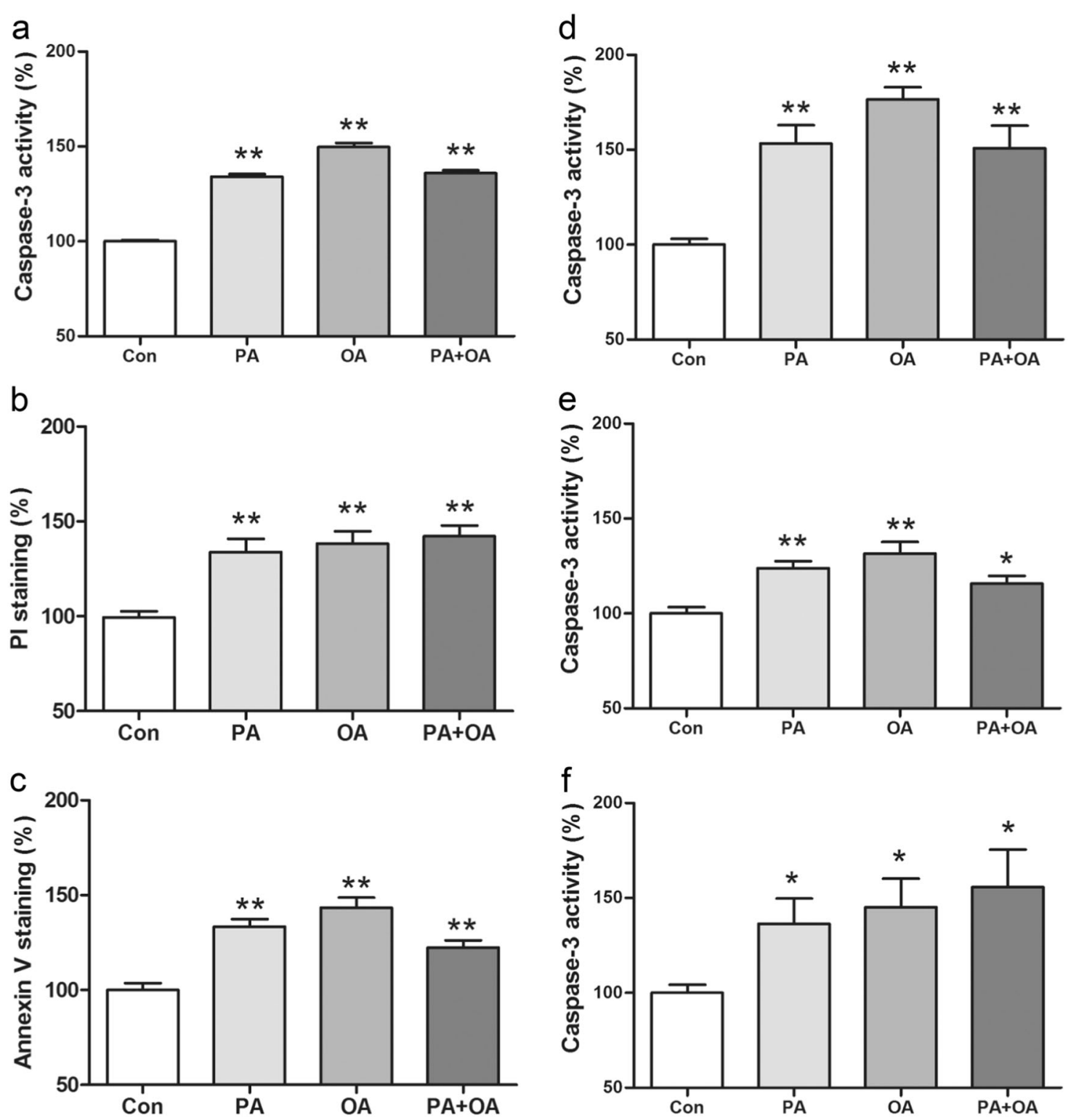

Fig. 1 Toxicities of PA, OA or the combination in human EndoC- $\beta \mathrm{H} 1$ beta cells by three different apoptosis tests a-c, and in human EndoC- $\beta \mathrm{H} 1 \mathrm{Pls} \mathbf{\mathbf { d }}$, isolated rat islets $\mathbf{e}$, and isolated human islets $\mathbf{f}$. Cells and islets were incubated for 2 days with PA $(500 \mu \mathrm{M}), \mathrm{OA}(500 \mu \mathrm{M})$ or a combination of both (500 $\mathrm{MM}$ each). Thereafter, toxicity was determined by measurements of caspase-3 activity $\mathbf{a}$, $\mathbf{d}$-f $\mathbf{f}$, propidium iodide (PI) staining $\mathbf{b}$ or annexin $\mathrm{V}$ staining $\mathbf{c}$. Data are means \pm SEM of 5-7 experiments from independent preparations in the EndoC- $\beta \mathrm{H} 1$ beta-cell experiments a-e. In the case of the rat islet studies, islets were isolated from six different animals $\mathbf{d}$ and in the case of the human islet studies from a single human donor $\mathbf{f} .{ }^{*} p<0.05,{ }^{* *} p$ $<0.01$ compared with untreated control cells.

treatment with the physiologically most prevalent saturated long-chain FFA PA $(\mathrm{C} 16: 0)(500 \mu \mathrm{M})$, as well as with the monounsaturated long-chain FFA OA (C18:1) $(500 \mu \mathrm{M})$. Independent confirmation for apoptotic cell death induced by the two FFAs was obtained in human EndoC- $\beta \mathrm{H} 1$ beta cells by toxicity analyses with the propidium iodide (Fig. 1b) and annexin V (Fig. 1c) staining tests. A combination of both FFAs $(500 \mu \mathrm{M}$ each) was also toxic, but an additive effect was not observed (Figs. 1a-f).

Toxicity was detected with FFAs having a minimum chain length of C16. Shorter FFAs were not toxic to human EndoC- $\beta \mathrm{H} 1$ beta cells. Thus, the medium-chain myristoleic acid (C14:1) $(100 \pm 3 \%, n=4)$ did not increase caspase- 3 activity as compared with the control situation $(100 \pm 2 \%, n=4)$. 


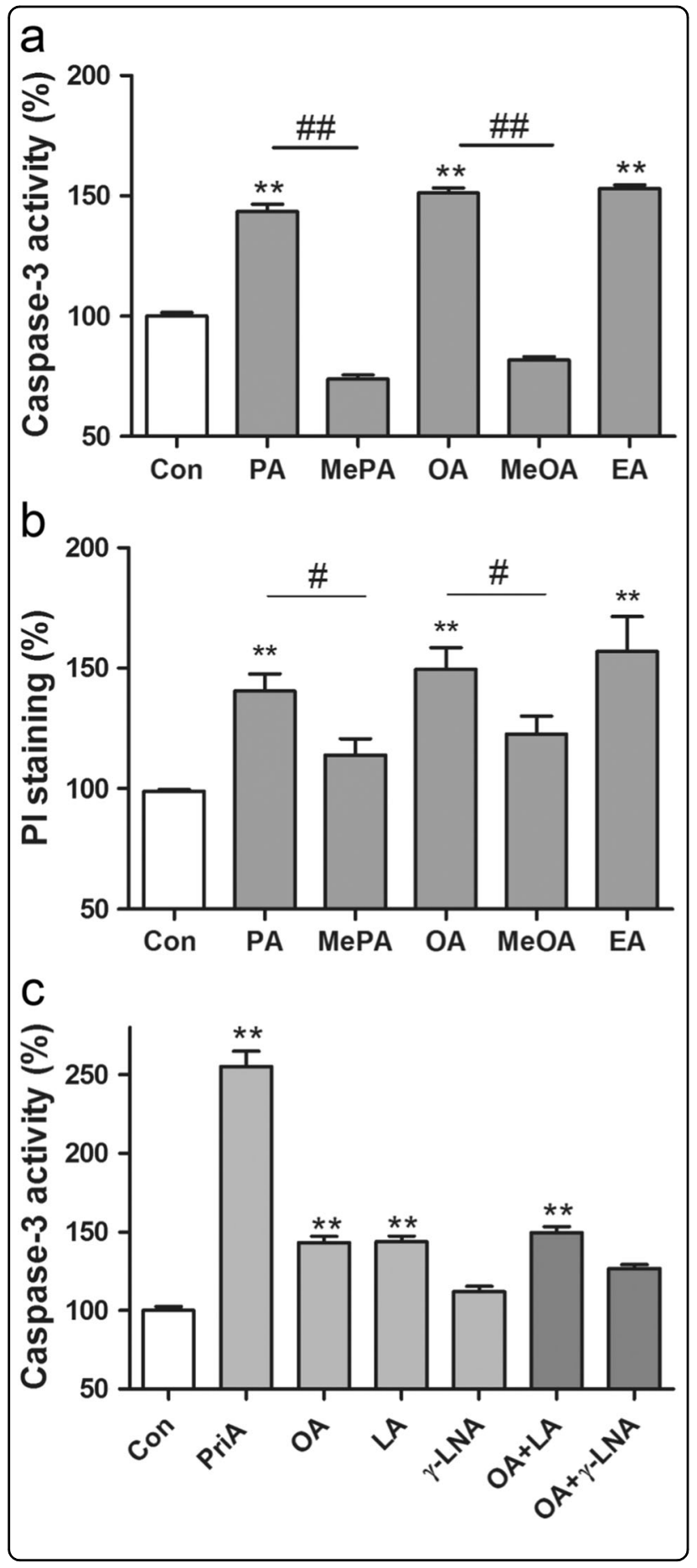

Not only PA and OA (cis-OA), but also EA (elaidic acid, the trans isomer of $\mathrm{OA}$; trans-OA) caused significant toxicity to human EndoC- $\beta \mathrm{H} 1$ beta cells in contrast to the non-metabolisable analogues methylated PA (MePA) and methylated OA (MeOA) (Figs. 2a, b). These results were obtained by the caspase- 3 apoptosis assay (Fig. 2a) and confirmed by the propidium iodide staining test (Fig. $2 b$ ).
Fig. 2 Toxicities of PA, MePA, OA, MeOA and EA (trans-OA) a-b and toxicities of the PUFAs LA (C18:2) and $\gamma$-LNA (C18:3) in comparison with the MUFA OA (C18:1) and to PriA in human EndoC- $\beta \mathrm{H} 1$ beta cells and their ability to antagonise the toxic effect of OA (C18:1) $\mathbf{c}$. EndoC$\mathrm{BH} 1$ beta cells were incubated for 2 days with PA, MePA, OA, MeOA and EA (trans-OA)(all FFAs $500 \mu \mathrm{M}$ ). Thereafter, caspase-3 activity a and propidium iodide fluorescence $\mathbf{b}$ were measured. In addition, EndoC$\beta \mathrm{H} 1$ beta cells were incubated for 2 days with the polyunsaturated fatty acids (PUFAs) LA $(500 \mu \mathrm{M}), \gamma$-LNA $(500 \mu \mathrm{M})$, and for comparison with the monounsaturated $\mathrm{OA}(500 \mu \mathrm{M})$, as well as the branchedchain PriA $(200 \mu M)$. Furthermore, combinations of OA with the PUFAs ( $500 \mu \mathrm{M}$ each) were incubated. Thereafter, caspase-3 activity was measured $\mathbf{c}$. Data are means \pm SEM of 5-8 independent experiments. ${ }^{* *} p<0.01$ compared with untreated control cells; ${ }^{*} p<0.05,{ }^{\# \#} p<0.01$ compared with the corresponding non-methylated fatty acid.

Further independent proof for apoptotic cell death caused by $\mathrm{PA}$ and $\mathrm{OA}$ has been provided by identification of apoptotic EndoC- $\beta \mathrm{H} 1$ beta-cell nuclei by electron microscopy (Supplementary Fig. S1).

Caspase- 3 was also significantly activated in human EndoC- $\beta \mathrm{H} 1$ beta cells after a 2-day treatment with the FFA linoleic acid (LA)(C18:2), but not with $\gamma$-linolenic acid $(\gamma$-LNA)(C18:3). Although LA showed a degree of toxicity similar to OA, $\gamma$-LNA was not significantly toxic to human EndoC- $\beta \mathrm{H} 1$ beta cells (Fig. $2 \mathrm{c}$ ). A significantly lower toxicity could also be documented for OA when combined with $\gamma$-LNA (C18:3), but not when combined with LA (C18:2) (Fig. 2c). The branched-chain fatty acid pristanic acid (PriA), which is exclusively metabolised in peroxisomal beta oxidation, was very toxic in these analyses (Fig. 2c).

The results of the present study document that the physiological MUFA OA is at least as toxic as the SFA PA to human beta cells. OA, the physiologically most abundant MUFA, which is even more prevalent in humans than $\mathrm{PA}^{11,12}$, cannot protect human beta cells against PA toxicity. And EA, the trans isomer of OA showed toxicity comparable to that of cis-OA.

As the non-metabolisable methylated analogues MePA and $\mathrm{MeOA}$ showed no significant toxicity to human EndoC- $\beta \mathrm{H} 1$ beta cells, the data support the conclusion that metabolism of the fatty acids is an indispensable prerequisite for their toxicity.

\section{Discussion}

Our data indicate that results obtained in previous lipotoxicity studies in rat insulin-producing cell lines cannot be simply extrapolated to primary pancreatic islets as $\mathrm{OA}$, in contrast to PA, was not or only minimally toxic and provided protection against the toxicity of SFAs such as PA in these insulin-producing rat cell lines ${ }^{10,15-17}$. In contrast, we document in the present study by three 
different apoptosis assays, as well as by electron microscopy the toxicity of OA and the inability of OA to reverse the toxic effect of PA. Beta cells of the permanent human EndoC- $\beta \mathrm{H} 1$ beta-cell line are a good substitute as they mirror the situation in rat, as well as in human primary pancreatic islets. On the other hand, OA in combination with PA did not show an additive toxic effect. Thus, a combined presence of SFAs and MUFAs in the diet, as well as in the physiological lipid composition in the human body is probably the optimum that can be achieved with respect to minimisation of lipotoxicity in the organism. Therefore, an emphasis on raising the proportion of MUFAs such as OA in diets too much and thereby creating an imbalance in favour of the MUFA component, through consumption of excessive amounts of vegetable oils such as olive oil, can be considered as counterproductive for beta-cell protection. Under this perspective, a vegetable oil with plenty of antioxidants but a limited content of OA might be the optimum that can be achieved when selecting a vegetable oil brand preparation, that is, for salad dressings.

PUFAs with more than two double bonds were less toxic and did provide protection against the toxicity of SFAs and MUFAs in human EndoC- $\beta \mathrm{H} 1$ beta cells. The proportion of PUFAs with more than two double bonds in human plasma is only around $3 \%^{11}$. So the amount of these PUFAs is insufficient to compensate for the toxicity of saturated and mono- and diunsaturated fatty acids in human beta cells.

Thus, the pancreatic beta-cell cannot be protected against the toxicity of SFAs through increased uptake of MUFAs. Therefore, it may be that the beta cell has to pay the price for the current emphasis on the consumption of a cardioprotective high MUFA diet. It is thus tempting to consider such a diet as a contributory factor to the continuing worldwide increase in the incidence of T2DM during recent decades (http://www.diabetesatlas.org/ resources/2015-atlas.html).

From studies in rat insulin-producing cell lines, it is known that hydrogen peroxide is the responsible toxic reactive species in PA-induced beta-cell toxicity ${ }^{18}$. This hydrogen peroxide is preferentially generated in the betaoxidation in the peroxisomes ${ }^{18}$, which has a preference for saturated and unsaturated fatty acids with chain lengths $>14 \mathrm{C}$-atoms ${ }^{10}$. This is exactly the preference for beta-cell lipotoxicity of long-chain fatty acids in human EndoC- $\beta \mathrm{H} 1$ beta cells that we document in the present study. Further support for the origin of the toxic hydrogen peroxide in the peroxisomal fatty acid metabolism comes from the observation that the branched-chain fatty acid PriA, which exclusively undergoes peroxisomal beta-oxidation ${ }^{10,19,20}$, was also shown to be toxic in the present study. PriA is the crucial reference substrate for peroxisomal betaoxidation $^{15}$. So it can be concluded that the different fatty acids, which have been found to be beta-cell toxic in the present study, are without exception substrates for the peroxisomal beta-oxidation ${ }^{15,19-21}$. Therefore, the present results support the concept of beta-cell lipotoxicity through peroxisomal stress ${ }^{15}$ that is mediated by hydrogen peroxide generation in the peroxisomal beta oxidation of the beta cells, which, at variance from virtually all other cell types, lack expression of the hydrogen peroxide inactivating enzyme catalase in the peroxisomes ${ }^{13,22}$.

The results obtained in the present study indicate that primary beta cells of rat and human origin, as well as human EndoC- $\beta \mathrm{H} 1$ beta cells differ from rat insulinproducing cell lines, in that these cell lines show no toxicity upon exposure to unsaturated fatty acids ${ }^{10,15-17}$ due to their inability to generate toxic hydrogen peroxide $^{10}$, as they apparently cannot metabolise unsaturated fatty acids in the peroxisomal beta-oxidation.

\section{Acknowledgements}

This work was supported by the European Union (Collaborative Project BetaBAT in the Framework Programme 7, grant agreement 277713). A.L. is a PhD student within the Research Training Group (GRK) 1947 BiOx of the German Research Council at the University of Greifswald. We are grateful to Maren Böger and Martin Wirth for their skilful technical assistance, and to Dr Guo Cai Huang for isolation of the human islets used in this study.

\section{Author details}

'Institute of Clinical Biochemistry, Hannover Medical School, Hannover, Germany. ${ }^{2}$ Institute of Experimental Diabetes Research, Hannover Medical School, Hannover, Germany. ${ }^{3}$ Institute of Medical Biochemistry and Molecular Biology, University Medicine Greifswald, Greifswald, Germany. ${ }^{4}$ Division of Diabetes and Nutritional Sciences, Faculty of Life Sciences and Medicine, King's College London, London, UK

\section{Competing interests}

The authors declare that they have no competing financial interests.

\section{Publisher's note}

Springer Nature remains neutral with regard to jurisdictional claims in published maps and institutional affiliations.

\section{Supplementary information}

The online version of this article (https://doi.org/10.1038/s41387-017-0005-x) contains supplementary material, which is available to authorized users.

Received: 23 February 2017 Revised: 6 September 2017 Accepted: 2 October 2017

Published online: 21 December 2017

\section{References}

1. Brown, J. M., Shelness, G. S. \& Rudel, L. L. Monounsaturated fatty acids and atherosclerosis: opposing views from epidemiology and experimental animal models. Curr. Atheroscler. Rep. 9, 494-500 (2007).

2. Shen, J. et al. Mediterranean dietary patterns and cardiovascular health. Annu. Rev. Nutr. 35, 425-449 (2015).

3. Keys, A. Coronary heart disease in seven countries. Circulation 41, 1-211 (1970).

4. de Lorgeril, M. \& Salen, P. The Mediterranean diet: rationale and evidence for its benefit. Curr. Atheroscler. Rep. 10, 518-522 (2008).

5. Lloyd-Jones, D. et al. American Heart Association Statistics C, Stroke Statistics S. Executive summary: heart disease and stroke statistics--2010 update: a report from the American Heart Association. Circulation 121, 948-954 (2010). 
6. Degirolamo, C. \& Rudel, L. L. Dietary monounsaturated fatty acids appear not to provide cardioprotection. Curr Atheroscler. Rep. 12, 391-396 (2010).

7. Garg, A. High-monounsaturated-fat diets for patients with diabetes mellitus: a meta-analysis. Am. J. Clin. Nutr. 67, 577S-582S (1998).

8. Ros, E. Dietary cis-monounsaturated fatty acids and metabolic control in type 2 diabetes. Am. J. Clin. Nutr. 78, 617S-625S (2003).

9. Chowdhury, R. et al. Association of dietary, circulating, and supplement fatty acids with coronary risk: a systematic review and meta-analysis. Ann. Intern. Med. 160, 398-406 (2014).

10. Gehrmann, W. et al. Antagonism between saturated and unsaturated fatty acids in ROS mediated lipotoxicity in rat insulin-producing cells. Cell. Physiol. Biochem. 36, 852-865 (2015).

11. Quehenberger, O. et al. Lipidomics reveals a remarkable diversity of lipids in human plasma. J. Lipid Res. 51, 3299-3305 (2010).

12. Iggman, D., Arnlov, J., Cederholm, T. \& Riserus, U. Association of adipose tissue fatty acids with cardiovascular and all-cause mortality in elderly men. JAMA Cardiol. 1, 745-753 (2016).

13. Gurgul-Convey, E., Mehmeti, I., Plötz, T., Jörns, A. \& Lenzen, S. Sensitivity profile of the human EndoC-betaH1 beta-cell line to proinflammatory cytokines. Diabetologia 59, 2125-2133 (2016).

14. Huang, G. C. et al. The development of new density gradient media for purifying human islets and islet-quality assessments. Transplantation 77, 143-145 (2004).
15. Gehrmann, W., Elsner, M. \& Lenzen, S. Role of metabolically generated reactive oxygen species for lipotoxicity in pancreatic beta-cells. Diabetes Obes. Metab. 12, 149-158 (2010).

16. Kharroubi, I. et al. Free fatty acids and cytokines induce pancreatic beta-cell apoptosis by different mechanisms: role of nuclear factorkappaB and endoplasmic reticulum stress. Endocrinology 145, 5087-5096 (2004).

17. Cunha, D. A. et al. Initiation and execution of lipotoxic ER stress in pancreatic beta-cells. J. Cell. Sci. 121, 2308-2318 (2008).

18. Elsner, M., Gehrmann, W. \& Lenzen, S. Peroxisome-generated hydrogen peroxide as important mediator of lipotoxicity in insulin-producing cells. Diabetes 60, 200-208 (2011).

19. Hiltunen, J. K., Karki, T., Hassinen, I. E. \& Osmundsen, H. Beta-oxidation of polyunsaturated fatty acids by rat liver peroxisomes. A role for 2,4-dienoylcoenzyme A reductase in peroxisomal beta-oxidation. J. Biol. Chem. 261 16484-16493 (1986).

20. Schulz, H. Beta oxidation of fatty acids. Biochim. Biophys. Acta 1081, 109-120 (1991).

21. Kunau, W. H., Dommes, V. \& Schulz, H. Beta-oxidation of fatty acids in mitochondria, peroxisomes, and bacteria: a century of continued progress. Prog. Lipid. Res 34, 267-342 (1995).

22. Lenzen, S. Oxidative stress: the vulnerable beta-cell. Biochem. Soc. Trans. 36 343-347 (2008). 